\title{
BIFURCATIONS OF PLANAR SLIDING HOMOCLINICS
}

\author{
JAN AWREJCEWICZ, MICHAL FEČKAN, AND PAWEL OLEJNIK
}

Received 31 August 2005; Accepted 11 September 2005

We study bifurcations from sliding homoclinic solutions to bounded solutions on $\mathbb{R}$ for certain discontinuous planar systems under periodic perturbations. Sufficient conditions are derived for such perturbation problems.

Copyright (C) 2006 Jan Awrejcewicz et al. This is an open access article distributed under the Creative Commons Attribution License, which permits unrestricted use, distribution, and reproduction in any medium, provided the original work is properly cited.

\section{Introduction}

We start from the planar discontinuous system

$$
\begin{array}{ll}
\dot{z}=f_{+}(z)+\varepsilon g(z, t, \varepsilon) & \text { for } y>1, \\
\dot{z}=f_{-}(z)+\varepsilon g(z, t, \varepsilon) & \text { for } y<1,
\end{array}
$$

where $z=(x, y) \in \mathbb{R}^{2}, f_{ \pm}, g$ are $C^{3}$-smooth, and $g$ is 1 -periodic in $t$. Here we set

$$
q_{ \pm}(z, t, \varepsilon)=f_{ \pm}(z)+\varepsilon g(z, t, \varepsilon) .
$$

We suppose the following conditions:

(i) $f_{-}(0)=0$, and $D f_{-}(0)$ has no eigenvalues on the imaginary axis,

(ii) there are two solutions $\gamma_{-}(s), \gamma_{+}(s)$ of $\dot{z}=f_{-}(z), y \leq 1$ defined on $\mathbb{R}_{-}=(-\infty, 0]$, $\mathbb{R}_{+}=[0,+\infty)$, respectively, such that $\lim _{s \rightarrow \pm \infty} \gamma_{ \pm}(s)=0$ and $\gamma_{ \pm}(s)=\left(x_{ \pm}(s), y_{ \pm}(s)\right)$ with $y_{ \pm}(0)=1, x_{-}(0)<x_{+}(0)$. Moreover, $f_{ \pm}(z)=\left(f_{ \pm 1}(z), f_{ \pm 2}(z)\right)$ with $f_{ \pm 1}(x, 1)>$ $0, f_{+2}(x, 1)<0$ for $x_{-}(0) \leq x \leq x_{+}(0)$. Furthermore, $f_{-2}(x, 1)>0$ for $x_{-}(0) \leq x<$ $x_{+}(0), f_{-2}\left(x_{+}(0), 1\right)=0$, and $\partial_{x} f_{-2}\left(x_{+}(0), 1\right)<0$.

Assumptions (i) and (ii) mean that (1.1) for $\varepsilon=0$ has a sliding homoclinic solution $\gamma$, created by $\gamma_{ \pm}$, to a hyperbolic equilibrium 0 . We are interested in the bifurcation of $\gamma$ to bounded solutions on $\mathbb{R}$ of (1.1) under the perturbation $\varepsilon g(z, t, \varepsilon)$. 
The plan of the paper is as follows. In Section 2, we study (1.1) by using functional methods based on [4] along with the implicit function theorem [5]. In Section 3, we generalize results of Section 2 to systems with multiple discontinuous levels. Final Section 4 is devoted to a concrete system of piece-vice linear systems with periodic perturbations.

Sliding periodic solutions of discontinuous differential equations are investigated in [1-3] with both analytical and numerical methods. Qualitative properties of discontinuous systems are studied in [6]. Bifurcations for planar discontinuous ordinary differential systems with small periodic perturbations from homoclinic solutions transversally intersecting levels of discontinuity are studied in [7] to generalize the well-known Melnikov method for a smooth case [4] to a discontinuous one. We note that bifurcations from sliding homoclinic solutions, studied in this paper, are different to $[4,7]$.

\section{Bifurcation result}

In this section, we find conditions under which $\gamma$ persists in (1.1) for $\varepsilon \neq 0$ small. For this purpose, we consider (1.1) as a system in $\mathbb{R}^{3}$ defined by

$$
\begin{array}{cc}
\dot{z}=f_{+}(z)+\varepsilon g(z, t, \varepsilon) & \text { for } y>1, \\
\dot{z}=f_{-}(z)+\varepsilon g(z, t, \varepsilon) & \text { for } y<1, \\
\dot{t}=1, &
\end{array}
$$

while on $y=1$ (cf. $[1,6])$, we consider the system

$$
\begin{aligned}
\dot{x}= & \frac{q_{+2}(x, 1, t, \varepsilon)}{q_{+2}(x, 1, t, \varepsilon)-q_{-2}(x, 1, t, \varepsilon)} q_{+1}(x, 1, t, \varepsilon) \\
& +\frac{q_{-2}(x, 1, t, \varepsilon)}{q_{-2}(x, 1, t, \varepsilon)-q_{+2}(x, 1, t, \varepsilon)} q_{-1}(x, 1, t, \varepsilon),
\end{aligned}
$$

where $q_{ \pm}=\left(q_{ \pm 1}, q_{ \pm 2}\right)$. We first study the system

$$
\begin{gathered}
\dot{z}=f_{-}(z)+\varepsilon g(z, t, \varepsilon) \quad \text { for } y \leq 1, \\
\dot{t}=1, \quad y(0)=1, \quad t(0)=\alpha, \quad s \leq 0 .
\end{gathered}
$$

Lemma 2.1. For any $\varepsilon$ small, there is a unique bounded solution $z_{-}(s, \varepsilon, \alpha)$ of $(2.3)$ on $\mathbb{R}_{-}$, which is near to $\gamma_{-}(s)$.

Proof. We consider the Banach space

$$
X=\left\{v=(x(s), y(s)) \in C_{b}\left(\mathbb{R}_{-}, \mathbb{R}^{2}\right) \mid y(0)=0\right\}
$$

with the usual sup-norm $\|\cdot\|$. We put $z=\gamma_{-}+v$ into (2.3) to get

$$
\begin{gathered}
\dot{v}=D f_{-}\left(\gamma_{-}(s)\right) v+\left\{f_{-}\left(\gamma_{-}(s)+v\right)-f_{-}\left(\gamma_{-}(s)\right)-D f_{-}\left(\gamma_{-}(s)\right) v\right\}+\varepsilon g\left(\gamma_{-}(s)+v, s+\alpha, \varepsilon\right), \\
v_{2}(0)=0,
\end{gathered}
$$


Jan Awrejcewicz et al. 3

where $v=\left(v_{1}, v_{2}\right)$. Next, the system

$$
\dot{v}=D f_{-}\left(\gamma_{-}(s)\right) v
$$

has an exponential dichotomy on $\mathbb{R}_{-}(\mathrm{cf} .[4])$, that is, there are positive constants $K, a$ and a projection $P: \mathbb{R}^{2} \rightarrow \mathbb{R}^{2}$ such that

$$
\begin{gathered}
\left\|V_{-}(s) P V_{-}(\theta)^{-1}\right\| \leq K e^{-a(s-\theta)} \quad \text { for } \theta \leq s \leq 0, \\
\left\|V_{-}(s)(\square-P) V_{-}(\theta)^{-1}\right\| \leq K e^{a(s-\theta)} \quad \text { for } s \leq \theta \leq 0,
\end{gathered}
$$

where $V_{-}(s), V_{-}(0)=\rrbracket$ is the fundamental matrix solution of (2.6). Moreover, since $\dot{\gamma}_{-}(s)$ solves (2.6), and it is bounded on $\mathbb{R}_{-}$, and $\dot{\gamma}_{-}(0)$ is transversal to the $x$-axis, we can sup-

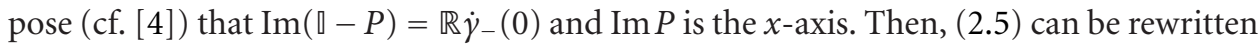
as a fixed point problem

$$
v(s)=\int_{-\infty}^{s} V_{-}(s) P V_{-}(\theta)^{-1} h(\theta) d \theta-\int_{s}^{0} V_{-}(s)(\square-P) V_{-}(\theta)^{-1} h(\theta) d \theta
$$

on the Banach space $X$, where

$$
h(\theta)=f_{-}\left(\gamma_{-}(\theta)+v(\theta)\right)-f_{-}\left(\gamma_{-}(\theta)\right)-D f_{-}\left(\gamma_{-}(\theta)\right) v(\theta)+\varepsilon g\left(\gamma_{-}(\theta)+v(\theta), \theta+\alpha, \varepsilon\right) .
$$

Since

$$
f_{-}\left(\gamma_{-}(\theta)+v\right)-f_{-}\left(\gamma_{-}(\theta)\right)-D f_{-}\left(\gamma_{-}(\theta)\right) v=O\left(|v|^{2}\right)
$$

for $\varepsilon$ small, we can solve (2.8) by using the implicit function theorem to obtain a unique small solution $v(s, \alpha, \varepsilon)$ of $(2.8)$, and so

$$
z(s, \alpha, \varepsilon)=\gamma_{-}(s)+v(s, \alpha, \varepsilon)
$$

solves (2.3). The proof is finished.

We put

$$
\varphi_{-}(\alpha, \varepsilon)=x(0, \alpha, \varepsilon)
$$

where

$$
z(s, \alpha, \varepsilon)=(x(s, \alpha, \varepsilon), y(s, \alpha, \varepsilon)) .
$$

Clearly, $\varphi_{-}(\alpha, 0)=x_{-}(0)$. Next, we consider (2.2) with the initial condition

$$
x(0)=\varphi_{-}(\alpha, \varepsilon) .
$$

If $h(x, s, \varepsilon)$ is the right-hand side of (2.2), then conditions (i) and (ii) imply that $h(x, s, \varepsilon)>$ 0 for any $x_{-}(0) \leq x \leq x_{+}(0)$ and $\varepsilon$ small. Then assumption (ii) gives the solvability of the equation

$$
q_{-2}\left(x\left(s_{+}(\alpha, \varepsilon)\right), 1, s_{+}(\alpha, \varepsilon)+\alpha, \varepsilon\right)=0
$$


4 Bifurcations of planar sliding homoclinics

for the function $s_{+}(\alpha, \varepsilon)>0$, where $x(s)$ solves (2.2) and (2.14). So, $s_{+}(\alpha, \varepsilon)$ is the time when the sliding motion of (2.2) is ending. We put

$$
\varphi_{+}(\alpha, \varepsilon)=x\left(s_{+}(\alpha, \varepsilon)\right) .
$$

Finally, we consider the initial value problem

$$
\begin{gathered}
\dot{z}=f_{-}(z)+\varepsilon g(z, t, \varepsilon) \quad \text { for } y \leq 1, \\
\dot{t}=1, \quad s \geq s_{+}(\alpha, \varepsilon), \\
z\left(s_{+}(\alpha, \varepsilon)\right)=\left(\varphi_{+}(\alpha, \varepsilon), 1\right), \quad t\left(s_{+}(\alpha, \varepsilon)\right)=s_{+}(\alpha, \varepsilon)+\alpha .
\end{gathered}
$$

That is the initial value problem

$$
\begin{gathered}
\dot{z}=f_{-}(z)+\varepsilon g(z, s+\alpha, \varepsilon) \quad \text { for } y \leq 1, \\
z\left(s_{+}(\alpha, \varepsilon)\right)=\left(\varphi_{+}(\alpha, \varepsilon), 1\right), \quad s \geq s_{+}(\alpha, \varepsilon) .
\end{gathered}
$$

We note that $\gamma_{+}(0)=\left(\varphi_{+}(\alpha, 0), 1\right)$ and we look for a solution $z$ of $(2.18)$ near to $\gamma_{+}(s-$ $\left.s_{+}(\alpha, \varepsilon)\right)=\omega_{+}(s)$. By taking

$$
z(s)=\omega_{+}(s)+\varepsilon w(s)
$$

in (2.18), we obtain

$$
\begin{gathered}
\dot{w}=D f_{-}\left(\omega_{+}(s)\right) w+\frac{1}{\varepsilon}\left\{f_{-}\left(\omega_{+}(s)+\varepsilon w\right)-f_{-}\left(\omega_{+}(s)\right)-D f_{-}\left(\omega_{+}(s)\right) \varepsilon w\right\} \\
+g\left(\omega_{+}(s)+\varepsilon w, s+\alpha, \varepsilon\right), \quad s \geq s_{+}(\alpha, \varepsilon) \\
w\left(s_{+}(\alpha, \varepsilon)\right)=\left(\psi_{+}(\alpha, \varepsilon), 0\right)
\end{gathered}
$$

where

$$
\psi_{+}(\alpha, \varepsilon)=\left(\varphi_{+}(\alpha, \varepsilon)-\varphi_{+}(\alpha, 0)\right) / \varepsilon .
$$

By shifting the time $s \leftrightarrow s+s_{+}(\alpha, \varepsilon), s \geq 0$ in (2.20), we obtain

$$
\begin{gathered}
\dot{w}=D f_{-}\left(\gamma_{+}(s)\right) w+\frac{1}{\varepsilon}\left\{f_{-}\left(\gamma_{+}(s)+\varepsilon w\right)-f_{-}\left(\gamma_{+}(s)\right)-D f_{-}\left(\gamma_{+}(s)\right) \varepsilon w\right\} \\
+g\left(\gamma_{+}(s)+\varepsilon w, s_{+}(\alpha, \varepsilon)+s+\alpha, \varepsilon\right), \quad s \geq 0, \\
w(0)=\left(\psi_{+}(\alpha, \varepsilon), 0\right) .
\end{gathered}
$$

We set

$$
\eta(\alpha, \varepsilon)=\left(\psi_{+}(\alpha, \varepsilon), 0\right)
$$

Now we study the problem

$$
\begin{gathered}
\dot{w}=D f_{-}\left(\gamma_{+}(s)\right) w+h(s), \\
w(0)=u,
\end{gathered}
$$


for $h \in C_{b}\left(\mathbb{R}_{+}, \mathbb{R}^{2}\right)$ and $u=\left(u_{1}, u_{2}\right) \in \mathbb{R}^{2}$. The system

$$
\dot{w}=D f_{-}\left(\gamma_{+}(s)\right) w
$$

has an exponential dichotomy on $\mathbb{R}_{+}$(cf. [4]), that is, there are positive constants $M, b$ and a projection $Q: \mathbb{R}^{2} \rightarrow \mathbb{R}^{2}$ such that

$$
\begin{gathered}
\left\|V_{+}(s) Q V_{+}(\theta)^{-1}\right\| \leq M e^{-b(s-\theta)} \quad \text { for } 0 \leq \theta \leq s, \\
\left\|V_{+}(s)(\square-Q) V_{+}(\theta)^{-1}\right\| \leq M e^{b(s-\theta)} \quad \text { for } 0 \leq s \leq \theta,
\end{gathered}
$$

where $V_{+}(s), V_{+}(0)=\llbracket$ is the fundamental matrix solution of (2.25). Moreover, since $\dot{\gamma}_{+}(s)$ solves (2.25) and it is bounded on $\mathbb{R}_{+}$, we can suppose (cf. [4]) that $\operatorname{Im} Q=\mathbb{R} \dot{\gamma}_{+}(0)$ and $\operatorname{Im}(\square-Q)$ is orthogonal to the line $\mathbb{R} \dot{\gamma}_{+}(0)$. On the other hand, condition (ii) implies that

$$
\dot{y}_{+}(0)=f_{-2}\left(x_{+}(0), y_{+}(0)\right)=f_{-2}\left(x_{+}(0), 1\right)=0 .
$$

So,

$$
\dot{\gamma}_{+}(0)=\left(\dot{x}_{+}(0), \dot{y}_{+}(0)\right)=\left(\dot{x}_{+}(0), 0\right) .
$$

Consequently, $Q$ is the orthogonal projection onto the $x$-axis. Let $\Gamma=\dot{\gamma}_{+}(0)^{\perp}$ be a nonzero orthogonal vector onto $\dot{\gamma}_{+}(0)$. Now, for simplicity, we can take $\Gamma=(0,1)$. So, $\operatorname{Im}(\square-Q)=$ $\mathbb{R} \Gamma$. We note that

$$
\mu(t)=V_{+}^{*}(s)^{-1} \Gamma
$$

is a basis of a space of bounded solutions on $\mathbb{R}_{+}$of the adjoint system (cf. [4])

$$
\dot{w}=-D f_{-}^{*}\left(\gamma_{+}(s)\right) w .
$$

We need the following result.

Lemma 2.2. Problem (2.24) has a bounded solution $w$ on $\mathbb{R}_{+}$if and only if

$$
\int_{0}^{+\infty}(h(s), \mu(s)) d s=-(\Gamma, u)=-u_{2}
$$

where $(\cdot, \cdot)$ is the usual scalar product on $\mathbb{R}^{2}$. Moreover, if condition (2.31) holds, then problem (2.24) has a unique bounded solution $w=w(u, h)$ on $\mathbb{R}_{+}$. Furthermore, there is a constant $c>0$ such that

$$
\|w(u, h)\| \leq c(\|h\|+|u|)
$$

where $\|\cdot\|$ is the sup-norm on $Y=C_{b}\left(\mathbb{R}_{+}, \mathbb{R}^{2}\right)$ and $|\cdot|$ corresponds to $(\cdot, \cdot)$.

Proof. A general form of a bounded solution of equation

$$
\dot{w}=D f_{-}\left(\gamma_{+}(s)\right) w+h(s)
$$


6 Bifurcations of planar sliding homoclinics

on $\mathbb{R}_{+}$is given by

$$
w(s)=c \dot{\gamma}_{+}(s)+\int_{0}^{s} V_{+}(s) Q V_{+}(\theta)^{-1} h(\theta) d \theta-\int_{s}^{+\infty} V_{+}(s)(\square-Q) V_{+}(\theta)^{-1} h(\theta) d \theta .
$$

Then using the initial condition $w(0)=u$, we get the equation

$$
u=c \dot{\gamma}_{+}(0)-\int_{0}^{+\infty}(\square-Q) V_{+}(\theta)^{-1} h(\theta) d \theta,
$$

which implies

$$
u_{2}=(u, \Gamma)=-\int_{0}^{+\infty}\left(V_{+}(s)^{-1} h(s), \Gamma\right) d s=-\int_{0}^{+\infty}\left(h(s), V_{+}^{*}(s)^{-1} \Gamma\right) d s=-\int_{0}^{+\infty}(h(s), \mu(s)) d s
$$

So, (2.31) is proved. On the other hand, if (2.31) holds, then (2.35) gives

$$
u_{1}=c \dot{x}_{+}(0)
$$

Consequently, the unique bounded solution of (2.24) on $\mathbb{R}_{+}$is given by

$$
w(s)=\frac{u_{1}}{\dot{x}_{+}(0)} \dot{\gamma}_{+}(s)+\int_{0}^{s} V_{+}(s) Q V_{+}(\theta)^{-1} h(\theta) d \theta-\int_{s}^{+\infty} V_{+}(s)(\square-Q) V_{+}(\theta)^{-1} h(\theta) d \theta .
$$

Then, (2.32) follows directly from (2.38). The proof is finished.

Let $S: Y \rightarrow Y$ be a projection defined by

$$
S h=h(s)-\int_{0}^{+\infty}\left[\left(h(\theta), \frac{\mu(\theta)}{\|\mu\|_{2}^{2}}\right) d \theta\right] \mu(s),
$$

where $\|u\|_{2}^{2}=\int_{0}^{+\infty} \mu(\theta)^{2} d \theta$. Then, (2.22) is splitted as follows

$$
\begin{gathered}
\dot{w}=D f_{-}\left(\gamma_{+}(s)\right) w+S\left[\frac{1}{\varepsilon}\left\{f_{-}\left(\gamma_{+}+\varepsilon w\right)-f_{-}\left(\gamma_{+}\right)-D f_{-}\left(\gamma_{+}\right) \varepsilon w\right\}\right. \\
\left.+g\left(\gamma_{+}+\varepsilon w, s_{+}(\alpha, \varepsilon)+s+\alpha, \varepsilon\right)\right], \\
w(0)=\left(\psi_{+}(\alpha, \varepsilon), 0\right), \\
\int_{0}^{+\infty}\left(\frac{1}{\varepsilon}\left\{f_{-}\left(\gamma_{+}(s)+\varepsilon w(s)\right)-f_{-}\left(\gamma_{+}(s)\right)-D f_{-}\left(\gamma_{+}(s)\right) \varepsilon w(s)\right\}\right. \\
\left.+g\left(\gamma_{+}(s)+\varepsilon w(s), s_{+}(\alpha, \varepsilon)+s+\alpha, \varepsilon\right), \mu(s)\right) d s=0 .
\end{gathered}
$$

By using Lemma 2.2 together with the implicit function theorem, we can solve (2.40) to obtain its solution

$$
w=w(\alpha, \varepsilon, s) .
$$


Then, by plugging it into (2.41), we arrive at a bifurcation equation

$$
\begin{aligned}
B(\alpha, \varepsilon)=\int_{0}^{+\infty}( & \frac{1}{\varepsilon}\left\{f_{-}\left(\gamma_{+}(s)+\varepsilon w(\alpha, \varepsilon, s)\right)-f_{-}\left(\gamma_{+}(s)\right)-D f_{-}\left(\gamma_{+}(s)\right) \varepsilon w(\alpha, \varepsilon, s)\right\} \\
& \left.+g\left(\gamma_{+}(s)+\varepsilon w(\alpha, \varepsilon, s), s_{+}(\alpha, \varepsilon)+s+\alpha, \varepsilon\right), \mu(s)\right) d s=0 .
\end{aligned}
$$

We have

$$
\bar{M}(\alpha)=B(\alpha, 0)=\int_{0}^{+\infty}\left(g\left(\gamma_{+}(s), s_{+}(\alpha, 0)+s+\alpha, 0\right), \mu(s)\right) d s=0
$$

Any simple root $\alpha_{0}$ of $\bar{M}(\alpha)$; that is, $\bar{M}\left(\alpha_{0}\right)=0$ and $\bar{M}^{\prime}\left(\alpha_{0}\right) \neq 0$, gives the solvability of $B(\alpha, \varepsilon)=0$ with respect to $\alpha=\alpha(\varepsilon)$ for any $\varepsilon$ small with $\alpha(0)=\alpha_{0}$.

On the other hand, from the definition of function $s_{+}(\alpha, \varepsilon)$ in $(2.15)$, we see that $\partial_{\alpha} s_{+}(\alpha, 0)=0$. So, simple roots of $\bar{M}(\alpha)$ are in one-to-one correspondence with simple roots of the function

$$
M(\beta)=\int_{0}^{+\infty}\left(g\left(\gamma_{+}(s), \beta+s, 0\right), \mu(s)\right) d s
$$

Summarizing we arrive at the following result.

Theorem 2.3. If there is a simple root $\beta_{0}$ of $M(\beta)$, that is, it holds that $M\left(\beta_{0}\right)=0$ and $M^{\prime}\left(\beta_{0}\right) \neq 0$, then homoclinic solution $\gamma$ bifurcates to a bounded solution on $\mathbb{R}$ of (1.1) with $\varepsilon \neq 0$ small.

\section{Generalization to multiple discontinuous systems}

The above approach to (1.1) can be generalized to cases when homoclinic orbit $\gamma(s)$ transversally crosses another curve of discontinuity. For simplicity, we suppose that such a discontinuity in (1.1) occurs at the level $y=1 / 2$, that is, in this section, we deal with the system

$$
\begin{array}{ll}
\dot{z}=f_{+}(z)+\varepsilon g(z, t, \varepsilon) & \text { for } y>1, \\
\dot{z}=f_{-}(z)+\varepsilon g(z, t, \varepsilon) & \text { for } \frac{1}{2}<y<1, \\
\dot{z}=F(z)+\varepsilon g(z, t, \varepsilon) & \text { for } y<\frac{1}{2},
\end{array}
$$

where $z=(x, y) \in \mathbb{R}^{2}, f_{ \pm}, F, g$ are $C^{3}$-smooth and $g$ is 1-periodic in $t$. We suppose the following conditions:

(a) $F(0)=0$ and $D F(0)$ has no eigenvalues on the imaginary axis,

(b) there are two solutions $\eta_{-}, \eta_{+}$of $\dot{z}=f_{-}(z), 1 / 2 \leq y \leq 1$ defined on $\left[a_{-}, 0\right],\left[0, a_{+}\right]$, $a_{-}<0<a_{+}$, respectively, such that $\eta_{ \pm}(s)=\left(\tilde{x}_{ \pm}(s), \tilde{y}_{ \pm}(s)\right)$ with $\tilde{y}_{ \pm}(0)=1$, $\tilde{y}_{ \pm}\left(a_{ \pm}\right)=1 / 2, \tilde{x}_{-}(0)<\tilde{x}_{+}(0), \tilde{x}_{-}\left(a_{-}\right)<\tilde{x}_{+}\left(a_{+}\right)$. Moreover, $f_{ \pm}(z)=\left(f_{ \pm 1}(z), f_{ \pm 2}(z)\right)$ with $f_{ \pm 1}(x, 1)>0, f_{+2}(x, 1)<0$ for $\tilde{x}_{-}(0) \leq x \leq \tilde{x}_{+}(0)$. Furthermore, $f_{-2}(x, 1)>0$ for $\tilde{x}_{-}(0) \leq x<\tilde{x}_{+}(0), f_{-2}\left(\tilde{x}_{+}(0), 1\right)=0$, and $\partial_{x} f_{-2}\left(\tilde{x}_{+}(0), 1\right)<0$. Finally, we suppose that $f_{-2}\left(\eta_{-}\left(a_{-}\right)\right)>0$ and $f_{-2}\left(\eta_{+}\left(a_{+}\right)\right)<0$, 
(c) there are two solutions $\tilde{\gamma}_{-}(s), \tilde{\gamma}_{+}(s)$ of $\dot{z}=F(z), y \leq 1 / 2$ defined on $\mathbb{R}_{-}=(-\infty, 0]$, $\mathbb{R}_{+}=[0,+\infty)$, respectively, such that $\lim _{s \rightarrow \pm \infty} \tilde{\gamma}_{ \pm}(s)=0$ and $\tilde{\gamma}_{ \pm}(0)=\eta_{ \pm}\left(a_{ \pm}\right)$. Moreover, $F(z)=\left(F_{1}(z), F_{2}(z)\right)$ with $F_{2}\left(\tilde{\gamma}_{-}(0)\right)>0$ and $F_{2}\left(\tilde{\gamma}_{+}(0)\right)<0$.

Again, assumptions (a), (b), and (c) imply that (3.1) for $\varepsilon=0$ has a sliding homoclinic solution $\tilde{\gamma}$, created by $\eta_{ \pm}$and $\tilde{\gamma}_{ \pm}$, to a hyperbolic equilibrium 0 . We study in this section bifurcation of $\tilde{\gamma}$ in system (3.1) for $\varepsilon \neq 0$ small. We can directly follow a method of Section 2. We first solve the equation

$$
q_{-2}\left(\tilde{\varphi}_{+}(\alpha, \varepsilon), 1, \alpha, \varepsilon\right)=0
$$

Since

$$
\begin{gathered}
q_{-2}\left(\tilde{x}_{+}(0), 1, \alpha, 0\right)=f_{-2}\left(\tilde{x}_{+}(0), 1\right)=0, \\
\partial_{x} q_{-2}\left(\tilde{x}_{+}(0), 1, \alpha, 0\right)=\partial_{x} f_{-2}\left(\tilde{x}_{+}(0), 1\right) \neq 0,
\end{gathered}
$$

we can solve (3.2) with $\tilde{\varphi}_{+}(\alpha, 0)=\tilde{x}_{+}(0)$. Next, we consider the initial value problem

$$
\begin{gathered}
\dot{z}=f_{-}(z)+\varepsilon g(z, t, \varepsilon) \quad \text { for } \frac{1}{2} \leq y \leq 1, \\
\dot{t}=1, \quad s \geq 0, \\
z(0)=\left(\tilde{\varphi}_{+}(\alpha, \varepsilon), 1\right), \quad t(0)=\alpha
\end{gathered}
$$

which has a unique solution

$$
\widetilde{z}(s, \alpha, \varepsilon)=(\tilde{x}(s, \alpha, \varepsilon), \tilde{y}(s, \alpha, \varepsilon)) .
$$

Then condition (b) implies that there is the smallest time $\tilde{s}_{+}(\alpha, \varepsilon)$ such that

$$
\tilde{y}\left(\widetilde{s}_{+}(\alpha, \varepsilon), \alpha, \varepsilon\right)=\frac{1}{2} .
$$

So, $\widetilde{s}_{+}(\alpha, \varepsilon)$ is the first hitting time for the level $y=1 / 2$ of the solution of (3.4). We set

$$
\xi(\alpha, \varepsilon)=\tilde{x}\left(\widetilde{s}_{+}(\alpha, \varepsilon), \alpha, \varepsilon\right) .
$$

Consequently, in order to study the bifurcation of $\tilde{\gamma}$, we need to show that the point $(\xi(\alpha, \varepsilon), 1 / 2)$ lies on the stable manifold of a unique small 1-periodic solution of (3.1). So we consider the initial value problem

$$
\begin{gathered}
\dot{z}=F(z)+\varepsilon g(z, t, \varepsilon), \\
\dot{t}=1, \\
z\left(\tilde{s}_{+}(\alpha, \varepsilon)\right)=\left(\xi(\alpha, \varepsilon), \frac{1}{2}\right), \quad t\left(\tilde{s}_{+}(\alpha, \varepsilon)\right)=\tilde{s}_{+}(\alpha, \varepsilon)+\alpha,
\end{gathered}
$$

that is the initial value problem

$$
\begin{gathered}
\dot{z}=F(z)+\varepsilon g\left(z, \tilde{s}_{+}(\alpha, \varepsilon)+s+\alpha, \varepsilon\right), \\
z(0)=\left(\xi(\alpha, \varepsilon), \frac{1}{2}\right), \quad s \geq 0 .
\end{gathered}
$$


We note $\tilde{\gamma}_{+}(0)=(\xi(\alpha, 0), 1 / 2)$. By taking

$$
z(s)=\tilde{\gamma}_{+}(s)+\varepsilon w(s)
$$

in (3.9), we get

$$
\begin{gathered}
\dot{w}=D F\left(\tilde{\gamma}_{+}(s)\right) w+\frac{1}{\varepsilon}\left\{F\left(\tilde{\gamma}_{+}(s)+\varepsilon w\right)-F\left(\tilde{\gamma}_{+}(s)\right)-D F\left(\tilde{\gamma}_{+}(s)\right) \varepsilon w\right\} \\
+g\left(\tilde{\gamma}_{+}(s)+\varepsilon w, \tilde{s}_{+}(\alpha, \varepsilon)+s+\alpha, \varepsilon\right), \quad s \geq 0, \\
w(0)=\left(\tilde{\psi}_{+}(\alpha, \varepsilon), 0\right)=\Psi_{+}(\alpha, \varepsilon),
\end{gathered}
$$

where

$$
\widetilde{\psi}_{+}(\alpha, \varepsilon)=(\xi(\alpha, \varepsilon)-\xi(\alpha, 0)) / \varepsilon
$$

Now we can repeat the above arguments of (2.22) to solve (3.11). So we again take

$$
\Gamma=\dot{\tilde{\gamma}}_{+}(0)^{\perp}=\left(\dot{\tilde{\gamma}}_{+2}(0),-\dot{\tilde{\gamma}}_{+1}(0)\right)
$$

The statement of Lemma 2.2 changes as follows.

Lemma 3.1. Problem

$$
\begin{gathered}
\dot{w}=D F\left(\tilde{\gamma}_{+}(s)\right) w+h, \\
w(0)=u
\end{gathered}
$$

has a bounded solution w on $\mathbb{R}_{+}$for a $h \in C_{b}\left(\mathbb{R}_{+}, \mathbb{R}^{2}\right)$ if and only if

$$
\int_{0}^{+\infty}(h(s), \tilde{\mu}(s)) d s+\left(\dot{\tilde{\gamma}}_{+}(0)^{\perp}, u\right)=0 .
$$

Moreover, if condition (3.15) holds, then problem (3.14) has a unique bounded solution $w=\tilde{w}(u, h)$ on $\mathbb{R}_{+}$. Furthermore, there is a constant $\tilde{c}>0$ such that

$$
\|\widetilde{w}(u, h)\| \leq \tilde{c}(\|h\|+|u|) .
$$

Here, $\tilde{\mu}$ is a bounded solution on $\mathbb{R}_{+}$of the adjoint linear equation

$$
\dot{w}=-D F\left(\tilde{\gamma}_{+}(s)\right)^{*} w
$$

with $w(0)=\Gamma$.

Condition (3.15) yields that instead of projection $S$ from Section 2, we take a mapping $\tilde{S}: \mathbb{R}^{2} \times Y \rightarrow Y$ defined by

$$
\tilde{S}(u) h=h-\int_{0}^{+\infty}\left[\left(h(\theta), \frac{\tilde{\mu}(\theta)}{\|\tilde{\mu}\|_{2}^{2}}\right) d \theta\right] \tilde{\mu}-\left(\dot{\tilde{\gamma}}_{+}(0)^{\perp}, u\right) \frac{\tilde{\mu}}{\|\tilde{\mu}\|_{2}^{2}} .
$$


Then we have

$$
\int_{0}^{+\infty}(\widetilde{S}(u) h(s), \tilde{\mu}(s)) d s+\left(\dot{\tilde{\gamma}}_{+}(0)^{\perp}, u\right)=0
$$

So we split (3.11) as follows:

$$
\begin{gathered}
\dot{w}=D F\left(\tilde{\gamma}_{+}(s)\right) w+\widetilde{S}\left(\Psi_{+}(\alpha, \varepsilon)\right)\left[\frac{1}{\varepsilon}\left\{F\left(\tilde{\gamma}_{+}(s)+\varepsilon w\right)-F\left(\tilde{\gamma}_{+}(s)\right)-D F\left(\tilde{\gamma}_{+}(s)\right) \varepsilon w\right\}\right. \\
\left.+g\left(\tilde{\gamma}_{+}(s)+\varepsilon w, \tilde{s}_{+}(\alpha, \varepsilon)+s+\alpha, \varepsilon\right)\right], \\
w(0)=\Psi_{+}(\alpha, \varepsilon), \\
\int_{0}^{+\infty}\left(\frac{1}{\varepsilon}\left\{F\left(\tilde{\gamma}_{+}(s)+\varepsilon w\right)-F\left(\tilde{\gamma}_{+}(s)\right)-D F\left(\tilde{\gamma}_{+}(s)\right) \varepsilon w\right\}\right. \\
\left.+g\left(\tilde{\gamma}_{+}(s)+\varepsilon w, \tilde{s}_{+}(\alpha, \varepsilon)+s+\alpha, \varepsilon\right), \tilde{\mu}(s)\right) d s \\
+\left(\dot{\tilde{\gamma}}_{+}(0)^{\perp}, \Psi_{+}(\alpha, \varepsilon)\right)=0 .
\end{gathered}
$$

By using Lemma 3.1, we can solve (3.20) to obtain its solution

$$
w=\widetilde{w}(\alpha, \varepsilon, s) .
$$

Then by inserting it into (3.21), we arrive at a bifurcation equation

$$
\begin{aligned}
\widetilde{B}(\alpha, \varepsilon)=\int_{0}^{+\infty}\left(\frac{1}{\varepsilon}\left\{F\left(\tilde{\gamma}_{+}(s)+\varepsilon \tilde{w}(\alpha, \varepsilon, s)\right)-F\left(\tilde{\gamma}_{+}(s)\right)-D F\left(\tilde{\gamma}_{+}(s)\right) \varepsilon \tilde{w}(\alpha, \varepsilon, s)\right\}\right. \\
\left.\quad+g\left(\tilde{\gamma}_{+}(s)+\varepsilon \tilde{w}(\alpha, \varepsilon, s), \tilde{s}_{+}(\alpha, \varepsilon)+s+\alpha, \varepsilon\right), \tilde{\mu}(s)\right) d s \\
+\left(\dot{\tilde{\gamma}}_{+}(0)^{\perp}, \Psi_{+}(\alpha, \varepsilon)\right)=0 .
\end{aligned}
$$

We have

$$
\widetilde{M}(\alpha)=\widetilde{B}(\alpha, 0)=\int_{0}^{+\infty}\left(g\left(\gamma_{+}(s), a_{+}+s+\alpha, 0\right), \tilde{\mu}(s)\right)+\dot{\tilde{\gamma}}_{+2}(0) \tilde{\psi}_{+}(\alpha, 0)=0,
$$

where we use that $\tilde{s}_{+}(\alpha, 0)=a_{+}$and

$$
\frac{1}{\varepsilon}\left\{F\left(\tilde{\gamma}_{+}(s)+\varepsilon \tilde{w}(\alpha, \varepsilon, s)\right)-F\left(\tilde{\gamma}_{+}(s)\right)-D F\left(\tilde{\gamma}_{+}(s)\right) \varepsilon \tilde{w}(\alpha, \varepsilon, s)\right\}=O(\varepsilon) .
$$

Any simple root $\alpha_{0}$ of $\widetilde{M}(\alpha)$ gives the solvability of $\widetilde{B}(\alpha, \varepsilon)=0$ with respect to $\alpha=\widetilde{\alpha}(\varepsilon)$ for any $\varepsilon$ small with $\tilde{\alpha}(0)=\alpha_{0}$.

Furthermore, from (3.6), we get

$$
f_{-2}\left(\eta_{+}\left(a_{+}\right)\right) \partial_{\varepsilon} \tilde{s}_{+}(\alpha, 0)+\partial_{\varepsilon} \tilde{y}\left(a_{+}, \alpha, 0\right)=0,
$$

while (3.7) and (3.12) give

$$
\widetilde{\psi}_{+}(\alpha, 0)=\partial_{\varepsilon} \xi(\alpha, 0)=f_{-1}\left(\eta_{+}\left(a_{+}\right)\right) \partial_{\varepsilon} \widetilde{s}_{+}(\alpha, 0)+\partial_{\varepsilon} \tilde{x}\left(a_{+}, \alpha, 0\right),
$$


which altogether imply

$$
\tilde{\psi}_{+}(\alpha, 0)=-f_{-1}\left(\eta_{+}\left(a_{+}\right)\right) \frac{\partial_{\varepsilon} \tilde{y}\left(a_{+}, \alpha, 0\right)}{f_{-2}\left(\eta_{+}\left(a_{+}\right)\right)}+\partial_{\varepsilon} \tilde{x}\left(a_{+}, \alpha, 0\right) .
$$

Next, we derive from (3.4) for

$$
w(s)=\partial_{\varepsilon} \tilde{z}\left(a_{+}, \alpha, 0\right)=\left(\partial_{\varepsilon} \tilde{x}\left(a_{+}, \alpha, 0\right), \partial_{\varepsilon} \tilde{y}\left(a_{+}, \alpha, 0\right)\right)
$$

the linear variational initial value problem

$$
\begin{gathered}
\dot{w}=D f_{-}\left(\eta_{+}(s)\right) w+g\left(\eta_{+}(s), s+\alpha, 0\right), \\
w(0)=\left(\partial_{\varepsilon} \tilde{\varphi}_{+}(\alpha, 0), 0\right) .
\end{gathered}
$$

But (3.2) implies

$$
\partial_{x} f_{-2}\left(\eta_{+}(0)\right) \partial_{\varepsilon} \tilde{\varphi}_{+}(\alpha, 0)+g_{2}\left(\eta_{+}(0), \alpha, 0\right)=0 .
$$

So instead of (3.30), we consider the linear initial value problem

$$
\begin{gathered}
\dot{w}=D f_{-}\left(\eta_{+}(s)\right) w+g\left(\eta_{+}(s), s+\alpha, 0\right), \\
w(0)=\left(-\frac{g_{2}\left(\eta_{+}(0), \alpha, 0\right)}{\partial_{x} f_{-2}\left(\eta_{+}(0)\right)}, 0\right) .
\end{gathered}
$$

Summarizing we arrive at the following result.

TheOrem 3.2. Let function $\widetilde{M}$ be given by (3.24) along with formulas (3.28), (3.29), and (3.32). If there is a simple root of $\widetilde{M}$, then homoclinic solution $\tilde{\gamma}$ bifurcates to a bounded solution on $\mathbb{R}$ of (3.1) with $\varepsilon \neq 0$ small.

\section{Example}

We present in this section an illustrative example. Let $a_{+}$be the unique (positive) solution of the equation

$$
e^{a_{+}}\left(1-a_{+}\right)=\frac{1}{2}
$$

We note that $a_{+} \sim 0.768039$. Then we set

$$
a=e^{a_{+}}\left(2-a_{+}\right) \sim 2.65554 .
$$

In this section, we consider system (3.1) with

$$
\begin{aligned}
& f_{+}(z)=\left\{\begin{array}{l}
\dot{x}=y, \\
\dot{y}=x-3 y,
\end{array} \quad f_{-}(z)=\left\{\begin{array}{l}
\dot{x}=y, \\
\dot{y}=2 y-x,
\end{array}\right.\right. \\
& F(z)=\left\{\begin{array}{l}
\dot{x}=-2 a y, \\
\dot{y}=-\frac{1}{2 a} x,
\end{array}\right.
\end{aligned}
$$


It is not difficult to see that now we have

$$
\begin{aligned}
& \eta_{+}(s)=\left\{\begin{array}{l}
e^{s}(2-s), \\
e^{s}(1-s),
\end{array} \quad \tilde{\gamma}_{+}(s)=\left\{\begin{array}{l}
e^{-s} a, \\
e^{-s} / 2,
\end{array}\right.\right. \\
& \tilde{\gamma}_{-}(s)=\left\{\begin{array}{l}
-e^{s} a, \\
e^{s} / 2,
\end{array} \eta_{-}(s)=\left\{\begin{array}{l}
-a e^{s-a_{-}}+\left(\frac{1}{2}+a\right) e^{s-a_{-}}\left(s-a_{-}\right), \\
\frac{1}{2} e^{s-a_{-}}+\left(\frac{1}{2}+a\right) e^{s-a_{-}}\left(s-a_{-}\right),
\end{array}\right.\right.
\end{aligned}
$$

where $a_{-} \sim-0.122043$ is the unique (negative) solution of the equation

$$
e^{a_{-}}+\left(\frac{1}{2}+a\right) a_{-}=\frac{1}{2}
$$

We note that $(a, 1 / 2)=\eta_{+}\left(a_{+}\right)$and system (3.32) has now the form

$$
\begin{gathered}
\dot{w}=\left(\begin{array}{cc}
0 & 1 \\
-1 & 2
\end{array}\right) w+\cos (s+\alpha)\left(\begin{array}{l}
0 \\
1
\end{array}\right), \\
w(0)=(\cos \alpha, 0) .
\end{gathered}
$$

After some computations, function (3.24) has now the form

$$
\begin{aligned}
\widetilde{M}(\alpha)= & \frac{a}{2}\left(\cos \left(a_{+}+\alpha\right)-\sin \left(a_{+}+\alpha\right)\right), \\
& +\frac{1}{4(1-a)} w_{2}\left(a_{+}\right)-\frac{1}{2} w_{1}\left(a_{+}\right),
\end{aligned}
$$

where $w(s)=\left(w_{1}(s), w_{2}(s)\right)$ solves (4.6), that is, we have

$$
\begin{gathered}
w_{1}\left(a_{+}\right)=\left(\cos \alpha+\frac{1}{2} \sin \alpha\right) e^{a_{+}}-\frac{1}{2}(\cos \alpha+\sin \alpha) e^{a_{+}} a_{+}-\frac{1}{2} \sin \left(a_{+}+\alpha\right), \\
w_{2}\left(a_{+}\right)=\frac{\cos \alpha}{2} e^{a_{+}}-\frac{1}{2}(\cos \alpha+\sin \alpha) e^{a_{+}} a_{+}-\frac{1}{2} \cos \left(a_{+}+\alpha\right) .
\end{gathered}
$$

Then, (4.7) takes the form

$$
\widetilde{M}(\alpha)=-0.441052 \cos \alpha-1.7501 \sin \alpha .
$$

Function (4.9) has two different simple roots over the period $2 \pi$. By applying Theorem 3.2 , we get the existence of two bounded solutions of (3.1) with (4.3) near to $\tilde{\gamma}$, which is homoclinic to a small hyperbolic $2 \pi$-periodic solution of (3.1) with (4.3).

\section{Acknowledgments}

The work of the first and third authors has been supported by the Polish Scientific Committee (KBN) under the Grant No. 4 T07A 031 28. The second author was supported by Grant VEGA-MS 1/2001/05. Finally, the second author thanks Flaviano Battelli for useful discussion concerning this paper. 


\section{References}

[1] J. Awrejcewicz, M. Fečkan, and P. Olejnik, On continuous approximation of discontinuous systems, Nonlinear Analysis 62 (2005), no. 7, 1317-1331.

[2] J. Awrejcewicz and P. Olejnik, Stick-slip dynamics of a two-degree-of-freedom system, International Journal of Bifurcation and Chaos 13 (2003), no. 4, 843-861.

[3] J. Awrejcewicz, P. Olejnik, and M. Fečkan, Sliding solution of a simple two degrees-of-freedom dynamical system with friction, Proceedings of 5th EUROMECH Nonlinear Dynamics Conference, Eindhoven, 2005, pp. 277-282.

[4] F. Battelli and C. Lazzari, Exponential dichotomies, heteroclinic orbits, and Mel'nikov functions, Journal of Differential Equations 86 (1990), no. 2, 342-366.

[5] K. Deimling, Multivalued Differential Equations, de Gruyter Series in Nonlinear Analysis and Applications, vol. 1, Walter de Gruyter, Berlin, 1992.

[6] R. I. Leine, D. H. Van Campen, and B. L. Van de Vrande, Bifurcations in nonlinear discontinuous systems, Nonlinear Dynamics 23 (2000), no. 2, 105-164.

[7] Y. K. Zou and T. Küpper, Melnikov method and detection of chaos for non-smooth systems, preprint, 2004.

Jan Awrejcewicz: Department of Automatics and Biomechanics, Technical University of Lodz, 1/15 Stefanowski Street, Lodz 90-924, Poland

E-mail address: awrejcew@p.lodz.pl

Michal Fečkan: Department of Mathematical Analysis and Numerical Mathematics, Comenius University, Mlynská dolina, 84248 Bratislava, Slovakia

E-mail address: michal.feckan@fmph.uniba.sk

Pawel Olejnik: Department of Automatics and Biomechanics, Technical University of Lodz, 1/15 Stefanowski Street, Lodz 90-924, Poland

E-mail address: olejnik@p.lodz.pl 


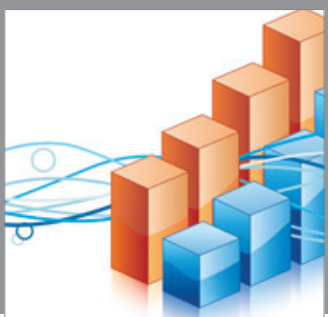

Advances in

Operations Research

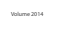

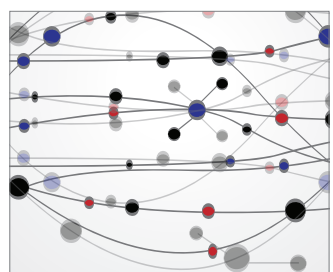

\section{The Scientific} World Journal
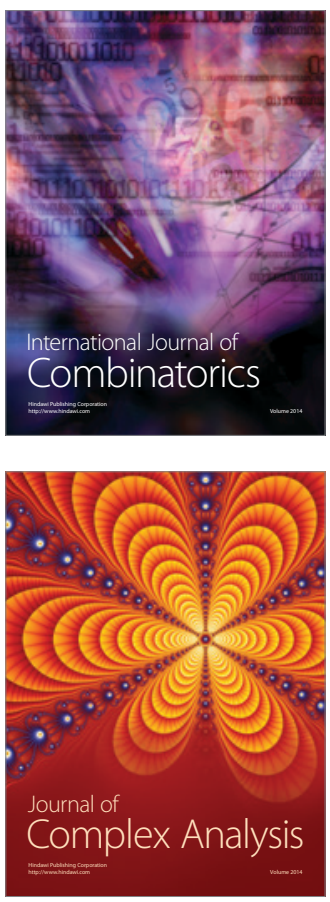

International Journal of

Mathematics and

Mathematical

Sciences
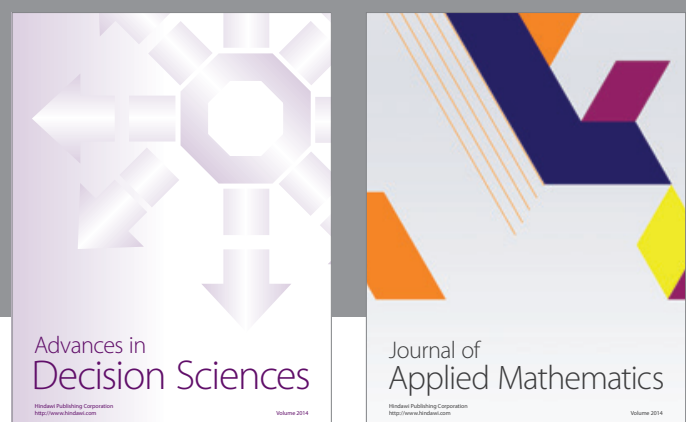

Journal of

Applied Mathematics
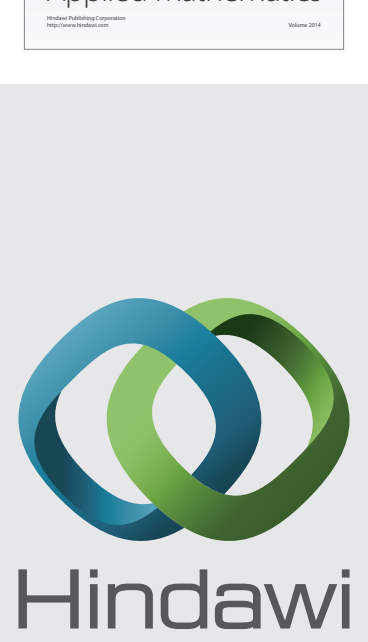

Submit your manuscripts at http://www.hindawi.com
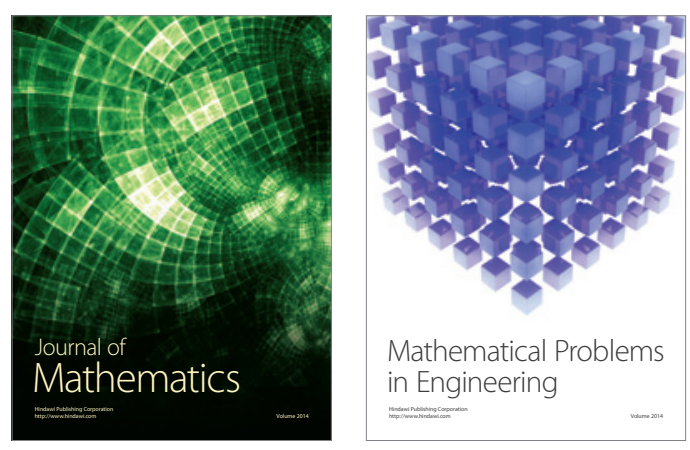

Mathematical Problems in Engineering
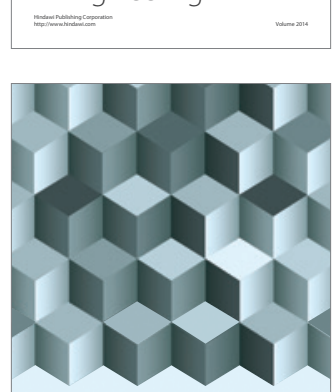

Journal of

Function Spaces
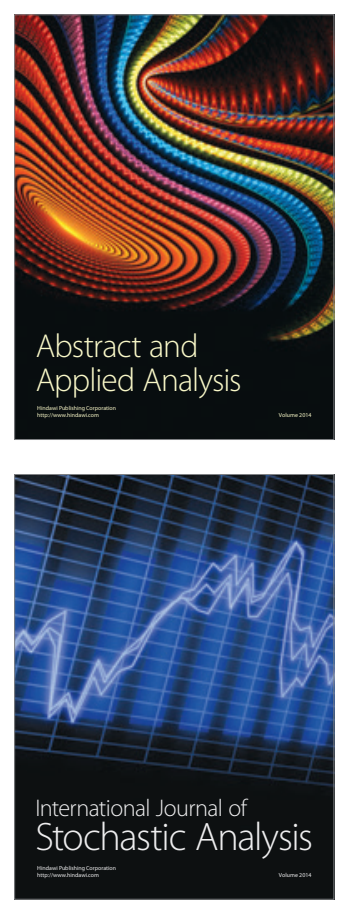

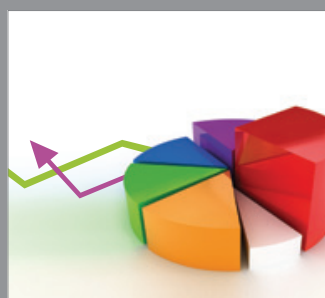

ournal of

Probability and Statistics

Promensencen
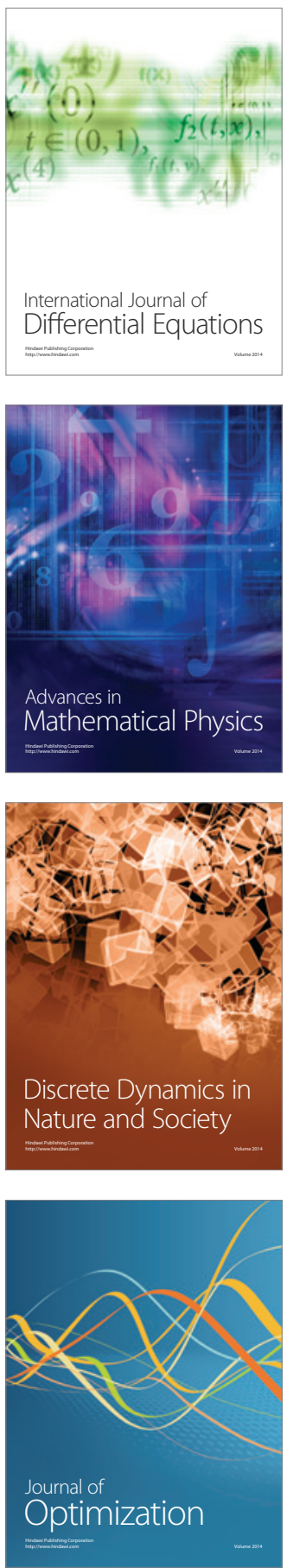